\title{
Bartonella henselae neuroretinitis in a patient without cat scratch
}

\author{
Claudia Montabone', Domizia Vecchio, ${ }^{1,2}$, Stela Vujosevic ${ }^{3}$, Stefano De Cillà ${ }^{3}$, Roberto Cantello,2 \\ ${ }^{1}$ Neurology Unit, Department of Translational Medicine, University of Piemonte Orientale, Novara 28100, Italy. \\ ${ }^{2}$ Interdisciplinary Research Center of Autoimmune Diseases (IRCAD), Department of Health Sciences, University of Piemonte Orientale, \\ Novara 28100, Italy. \\ ${ }^{3}$ Ophthalmology Unit, Department of Health Sciences, Azienda Ospedaliera Universitaria Maggiore della Carità, Novara 28100, Italy.
}

Correspondence to: Dr. Domizia Vecchio, Neurology Unit, Department of Translational Medicine, University of Piemonte Orientale, Corso G. Mazzini 18, Novara 28100, Italy.E-mail: domizia.vecchio@gmail.com

How to cite this article: Montabone C, Vecchio D, Vujosevic S, De Cillà S, Cantello R. Bartonella henselae neuroretinitis in a patient without cat scratch. Neuroimmuno/ Neuroinflammation 2019;6:7. http://dx.doi.org/10.20517/2347-8659.2019.09

Received: 5 Mar 2019 First Decision: 26 Mar 2019 Revised: 2 Apr 2019 Accepted: 2 Apr 2019 Published: 24 Apr 2019

Science Editor: Athanassios P. Kyritsis Copy Editor: Cai-Hong Wang Production Editor: Huan-Liang Wu

\begin{abstract}
Neuroretinitis is characterized by optic disc swelling with macular star, and affects $1 \%-2 \%$ of the patients with Bartonella henselae infection. This condition needs to be considered in the differential diagnosis of unilateral optic neuropathy in young adults. A 44-year-old woman presented with a progressive visual loss in right eye that was described as a central scotoma with altered color perception. Medical history was negative except for Hashimoto's thyroiditis. The examination evidenced a relative afferent pupillary defect in right eye and marked papillary oedema involving the macular region. Brain and orbits magnetic resonance imaging were normal, and fundoscopy showed star-shaped hard exudates. Autoimmune and infective screening revealed $\lg M$ and $\lg G$ antibodies against Bartonella henselae, suggesting for recent cat-scratch disease. She was treated with high-dose intravenous steroids and doxycycline. One month later she fully recovered, and she had no relapses. We diagnosed a Bartonella henselae neuroretinitis (finally the patient recalled she had stroked stray cats, not being scratched). In conclusion time course and absence of pain differentiate neuroretinitis from other optic neuropathies. Fundoscopic image of macular star is a clue for diagnosis, and visual recovery is usually excellent.
\end{abstract}

Keywords: Neuroretinitis, optic neuropathy, bartonella henselae, cat-scratch disease

\section{INTRODUCTION}

Neuroretinitis is a rare inflammatory optic neuropathy with direct involvement or autoimmune activation against the optic nerve. The inflammation of the optic disc vasculature causes exudation of fluid into the

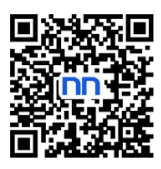


peripapillary retina, taking a characteristic macular star shape. Usual presentation is monocular central visual loss with discromatopsia and papillitis on funduscopic evaluation ${ }^{[1]}$.

The differential diagnosis includes inflammatory optic neuropathies such as sarcoidosis, systemic lupus erythematosus, Behçet syndrome, inflammatory bowel disease, and paraneoplastic syndromes ${ }^{[2]}$. Other causes are infections: herpetic viruses, HIV, West Nile virus and rarely flu or mumps, toxoplasmosis and toxocariasis, syphilis, Lyme disease, tuberculosis, rickettsiosis, and cat-scratch disease (CSD ${ }^{[3]}$. CSD is related to Bartonella species and transmitted through skin lesions after contact with infected animals ${ }^{[4]}$. A contact with cats is reported in $90 \%$ of CSD, and scratches or bites in $60 \%$ of the patients ${ }^{[5]}$.

Typical CSD presentation is a small papule at the inoculation site, followed by regional and systemic lymphadenopathy and fever. Ocular involvement occurs in 5\%-10\% of the patients, mostly presenting Parinaud syndrome (fever, regional lymphadenopathy, and follicular conjunctivitis). Neuroretinitis occurs in $1 \%-2 \%$ of the patients ${ }^{[6]}$, and other neurological manifestations are meningoencephalitis, myelitis, and acute polyneuropathies ${ }^{[7,8]}$.

Neuroretinitis is characterized by subretinal fluid, retinal thickening, and hyper-reflective exudates in the outer plexiform layer ${ }^{[9]}$ at optical coherence tomography. Fluorescein angiography shows fluid leakage, due to increased vessel permeability.

Currently, antibiotic therapy for Bartonella complicated infection with doxycycline and rifampin is recommended, either alone or in combination with corticosteroids. Habot-Wilner et al. ${ }^{[10]}$ showed a significant visual acuity improvement in those patients treated with antibiotics and corticosteroids if compared to those receiving only antibiotics. In the majority of cases no further progression of ocular inflammation was observed after therapy, and visual acuity recovered after 1-4 weeks ${ }^{[11]}$.

\section{CASE REPORT}

A 44-year-old woman presented to the Emergency Department with a progressive visual loss and altered color perception in the right eye, started one week before with no ocular pain, headache, dizziness, nausea or vomiting.

Her past medical history included Hashimoto's disease, treated with hormonal replacement therapy in the last 10 years. Particularly, the patient denied previous neurological or visual deficit. Her familial history was negative for neurological, vascular or autoimmune disease, and both her parents suffered from colorectal cancer. About two weeks before, she had fever and was treated with amoxicillin and clavulanic acid (875/125 mg b.i.d) for otitis. She reported a mild indolent cervical lymphadenopathy occurred 5-7 days after fever, and denied any cutaneous manifestation. Routine blood examinations were normal, including reactive $\mathrm{C}$ protein $(0.05$ $\mathrm{mg} / \mathrm{dL}, \mathrm{N} . \mathrm{V} .<1.00 \mathrm{mg} / \mathrm{dL}$ ). She had neither fever, night sweats, weight loss or other constitutional symptoms nor lymphnod involvement at the examination.

\section{Ophthalmological findings}

Ophthalmological examination revealed severely compromised visual acuity $(1 / 10$ in right eye vs. 8/10 in left eye), and computerized visual field testing evidenced a temporal loss in right eye. Ocular tonometry was normal. At the slit lamp the anterior segment was regular with normal pupillary reflexes, but there was a right relative afferent pupillary defect. On that side, fundoscopy showed marked papillary oedema involving macular region, hard retinal exudates with appearance of macular star, increased retinal vessel tortuosity and arteriovenous nicking. Left eye fundoscopy showed milder signs of inflammation with moderate edema. A fluorescein angiography confirmed the presence of retinal edema with leakage and optic coherence tomography (OCT) showed retinal thickening with initial exudates within the outer plexiform layer [Figure 1]. 

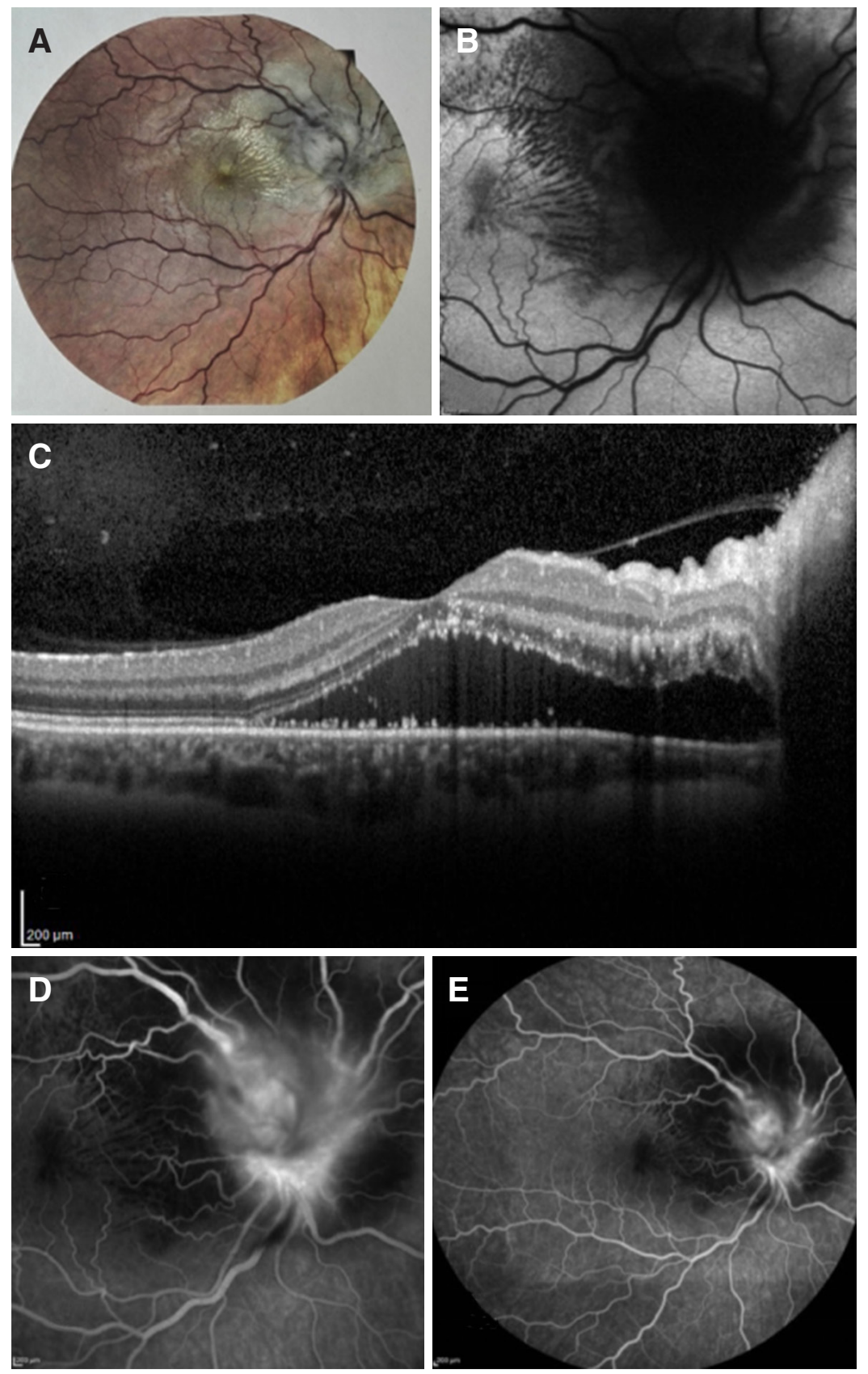

Figure 1. Instrumental imaging of right eye at symptoms presentation. A: colorful fundus photography; B: funduscopic scan with retinal exudates organizing in macular star appearance; C: OCT of right eye showing retinoic edema with peripapillary fluid; D: fluorescein angiography showing leakage of vessels from inflamed right optic disc; E: indocyanine green angiography

\section{Neurological findings}

A brain tomography was immediately performed to exclude an intracranial mass and one week later she had a magnetic resonance imaging (MRI) of brain and optic nerves, that resulted normal. Visual evoked potentials (VEP) showed a severe right delay of conduction (P100 latency was $123 \mathrm{~ms}$ ) [Figure 2].

Since the ophthalmological pattern was highly suggestive for an inflammatory neuroretinitis, a full blood analysis for autoimmune and infective causes was performed. Erythrocyte sedimentation rate was mildly elevated (38 mm/h N.V. 0-15), and serologic test for herpetic viruses including cytomegalovirus and Epstein Barr showed immunization from previous infections. Serology for toxoplasmosis, syphilis, Lyme disease and 


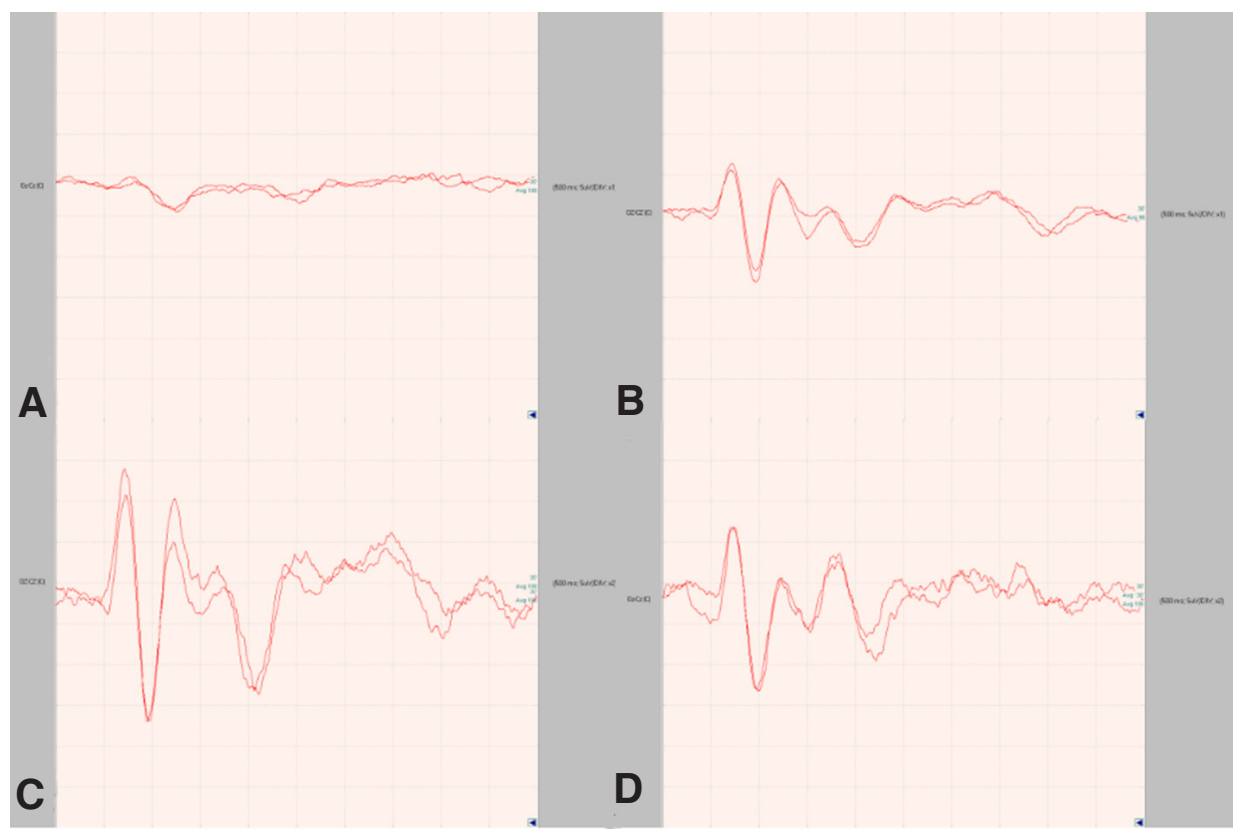

Figure 2. Visual evoked potential. A: right eye VEP at symptoms presentation (P100 latency $123 \mathrm{~ms}$ ); B: left eye (P100 latency $86 \mathrm{~ms})$; C: right eye VEP after one month; D: left eye VEP after one month

Toxocara canis were negative. Serology for Bartonella infections showed previous infection from Bartonella quintana (IgM antibody titer assay was not performed because of negative immunofluorescence, IgG titer was 1:128 that was at upper limit for positivity in our laboratory) and recent infection from Bartonella henselae with both IgM and IgG (IgM titer was 1:40 N.V. < 20, IgG was 1: 512 N.V. < 64).

\section{Treatment and follow up}

A high dose intravenous steroid regimen (methylprednisolone $1000 \mathrm{mg} / \mathrm{die}$ for 3 days) was started, followed by oral steroid tapering for one month. According to an infectiology consultation, antibiotic therapy with doxycycline $100 \mathrm{mg}$ b.i.d for two weeks was started.

One month later the patient fully recovered the visual acuity (10/10 in both eyes). OCT and fluorescein angiography showed marked reduction of optic disk swelling and disappearance of retinal exudates. VEP returned to normal [Figure 3]. Six months later, the patient doesn't complain any visual deficit, as confirmed at ophthal mologic instrumental evaluation. Neurologic examination is normal.

At that time, the patient was asked about previous contact with potentially infected animals, as she didn't own domestic animals. She recalled having handled stray cats during previous summer holidays (about one month before visual symptoms started), but she denied having been scratched.

\section{DISCUSSION}

We presented a young woman with a unilateral loss of vision caused by a CSD isolated neuroretinitis with mild constitutional symptoms and no history of scratch. Neurological examination, brain MRI and VEP did not give a clue for this diagnosis, whereas ophthalmological results suggested the possibility of an infection. The prompt treatment with antibiotics and steroids brought a complete resolution of the vision deficit.

This case represents a clinical rarity, first because of the diagnosis of CSD without cat scratch. Notably, CSD diagnosis requires the presence of at least 3 of 4 criteria including: a history of traumatic cat exposure, a positive test for CSD antibody, regional lymphadenopathies, and serum analysis excluding concurrent 

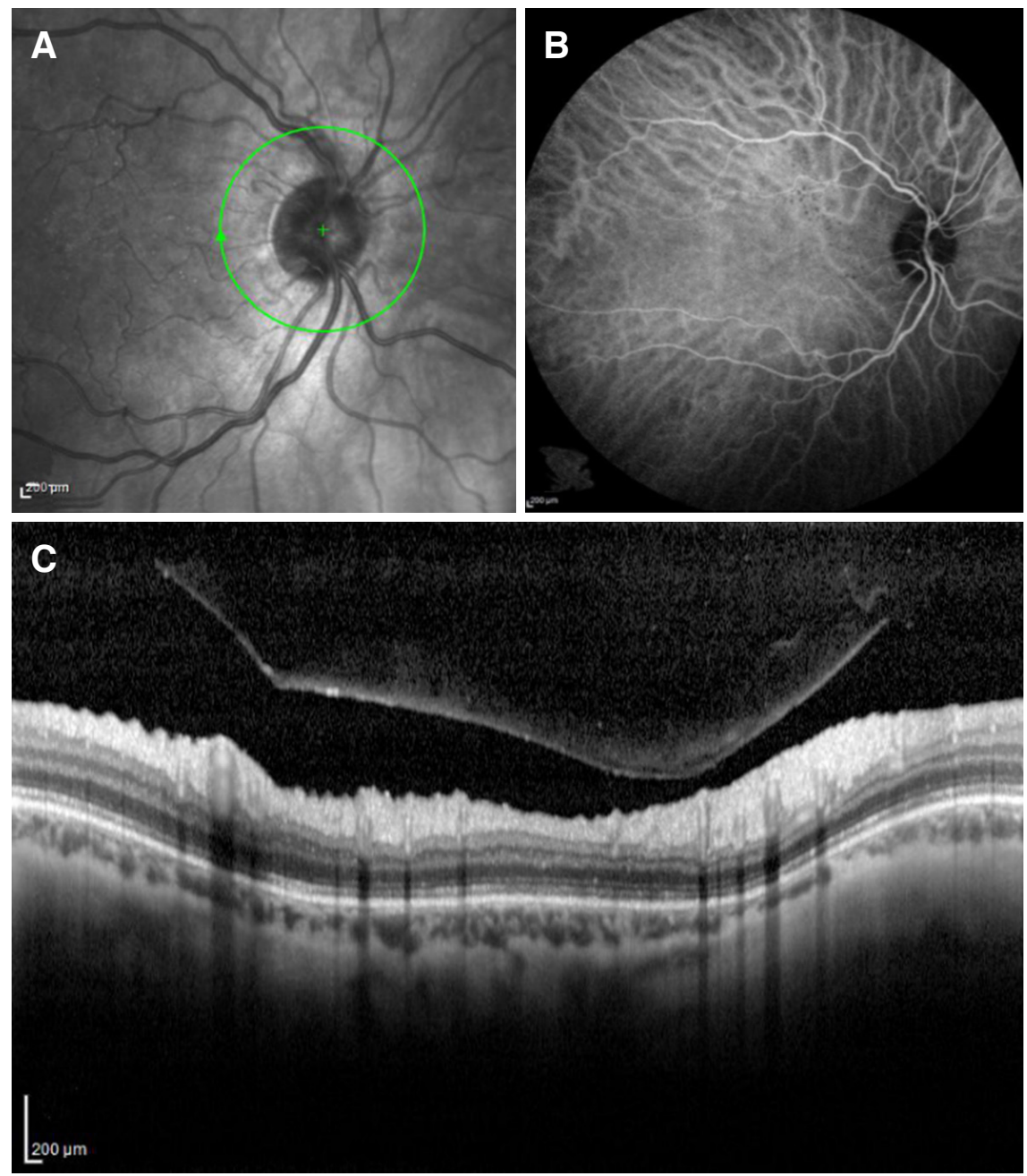

Figure 3. Instrumental imaging of right eye at one month, after treatment. A: funduscopic scan no retinal exudates; B: fluorescein angiography showing resolution of leakage; C: OCT of right eye showing resolution of edema

causes $^{[12,13]}$. Only 2 criteria were fulfilled in our case. Celiker et al ${ }^{[14]}$ reported three cases of isolated CSD neuroretinitis without contact with cats, and only $26 \%$ of patients recalled a history of cat scratch or bite in a case series of 19 patients with ocular bartonellosis ${ }^{[15]}$. This could be related to animal transmission by feces, that host Bartonella for several days and could infect man through an open wound or mucosae. Moreover, direct ocular infection is possible through the conjunctiva by eye rubbing, contaminated cosmetics or contact lenses ${ }^{[16]}$.

Our diagnosis of CSD neuroretinitis relied on the typical hallmarks for this condition: absence of pain, progressive course of visual loss in few days, initial bilateral involvement, and no altitudinal visual deficit. Time interval of 6 weeks between presumed exposition to infected animal and initial symptoms is highly suggestive $^{[17]}$. Instrumental findings were also indicative for neuroretinitis: i.e., OCT scan with subretinal fluid (as happens at very early stage with direct ophthalmoscopy or fluorescein angiography still normal) ${ }^{[18]}$, and the presence of macular star (pathognomonic of neuroretinitis). We also performed fluorescein angiography to exclude retinal arteriolar occlusions, a complication of ocular bartonellosis associated with very poor visual recovery $^{[19]}$.

Serological analysis confirmed our suspicion with both IgM and IgG for Bartonella henselae. This immunofluorescence assay has good specificity for $\operatorname{IgM}(87 \%-96 \%)$ and $\operatorname{IgG}(69 \%)$ although sensitivity is 
greater for IgG (88\%-98\%) compared to IgM $(50 \%-62 \%)^{[20]}$. False positives due to cross-reaction are highly improbable being about 2\%-5\% for Epstein Barr/Cytomegalovirus, and 20\%-50\% for Coxiella burnetii/ non-henselae bartonellosis (very rare in our country) ${ }^{[21]}$. IgM presence allows to date back infection to the previous 3 months, whereas $\operatorname{IgG}$ remains detectable in the $25 \%$ of patients one year later.

Finally, we observed full clinical and instrumental recovery after specific antibiotic and steroid therapy. In a retrospective study on $74 \mathrm{CSD}$ patients with ocular disease, 44 were treated with antibiotics alone, 17 with antibiotics and steroids (both intravenous high dose metil-prednisolone or oral prednisone), 2 with steroids alone and 11 patients didn't receive any treatment. Visual acuity improvement occurred in 50\% of the patients treated with antibiotics alone, and in $87.5 \%$ treated with both antibiotics and corticosteroids ${ }^{[10]}$.

The combination of doxycycline and steroids shorten the course of disease and accelerate visual recovery compared to natural history of illness in our case ${ }^{[22]}$. No side effects were reported.

CSD neuroretinitis is a rare clinical condition that must be considered in young patient with rapidly progressive unilateral visual loss. It requires a prompt neuro-ophthalmological evaluation in order to establish the correct diagnosis and start an early specific treatment.

\section{DECLARATIONS}

\section{Authors' contributions}

Made substantial contributions to realization of this case report: Montabone C, Vecchio D, Vujosevic S Reviewed the case as well as the manuscript, providing substantial contribution to discussion and conclusion: De Cillà S, Cantello R

\section{Availability of data and materials}

Not applicable.

\section{Financial support and sponsorship}

None.

\section{Conflicts of interest}

All authors declared that there are no conflicts of interest.

\section{Ethical approval and consent to participate}

The patient gave written informed consent.

\section{Consent for publication}

The patient gave written informed consent for publication. All clinical information submitted is anonymous and the patient is not recognizable from the iconographic material.

\section{Copyright}

(C) The Author(s) 2019.

\section{REFERENCES}

1. Purvin V, Sundaram S, Kawasaki A. Neuroretinitis: review of the literature and new observations. J Neuroophtalmol 2011;31:58-68.

2. Touitou V, LeHoang P. Diagnostic approach in optic neuropathy. Rev Neurol (Paris) 2012;168:691-6.

3. Kahloun R, Abroug N, Ksiaa I, Mahmoud A, Zeghidi H, et al. Infectious optic neuropathies: a clinical update. Eye Brain 2015; 7:59-81.

4. Mabra D, Yeh S, Shantha JG. Ocular manifestations of bartonellosis. Curr Opin Ophtalmol 2018;29:582-7.

5. Cunningham ET, Kochner JE. Ocular bartonellosis. Am J Opthalmol 2000;130: 340-9.

6. Murakami K, Tsukahara M, Tsuneoka H, Iino H, Ishida C, et al. Cat scratch disease: analysis of 130 sieropositive cases. J Infect 
Chemother 2002;8:349-52.

7. Canneti B, Cabo-López I, Puy-Núñez A, García García JC, Cores FJ, et al. Neurological presentations of bartonella henselae infection. Neurol Sci 2019;40:261-8.

8. Samarkos M, Antoniadou V, Vaiopoulos AG, Psichogiou M. Encephalopathy in an adult with cat-scratch disease. BMJ Case Rep 2018;5:2018.

9. Habot-Wilner Z, Zur D, Goldstein M, Goldenberg D, Shulman S. Macular findings on optical coherence tomography in cat-scratch disease neuroretinitis. Eye (Lond) 2011;25:1064-8.

10. Habot-Wilner Z, Trivizki O, Goldstein M, Kesler A, Shulman S, et al. Cat-stratch disease: ocular manifestations and treatment outcome. Acta Ophtalmol 2018: 96:e524-32.

11. Rolain JM, Brouqui P, Koehler JE, Maguina C, Dolan MJ, et al. Recommendations for treatment of human infections caused by Bartonella species. Antimicrob Agents Che-mother 2004; 48:1921-33.

12. Carithers HA. An overview based on a study of 1,200 patients. Am J Dis Child 1985;139:1124-33.

13. Margileth AM. Sorting out the causes of lymphadenopathy. Contemp Pediatr 1995;12:23.

14. Celiker H, Kazokoglu H, Eraslan M, Cerman E, Karabas L. Bartonella henselae neuroretinitis in patients without cat scratch. Jpn J Infect Dis 2018;71:397-401.

15. Tan CL, Fhun LC, Tai EL, Abdul Gani NH, Muhammed J, et al. Clinical profile and visual outcome of ocular bartonellosis in Malaysia. J Trop Med 2017;2017:7946123.

16. Foil L, Andress E, Freeland RL, Roy AF, Rutledge R, et al. Experimental infection of domestic cats with Bartonella henselae by inoculation of Ctenocephalides felis (Siphonaptera: Pulicidae) feces. J Med Entomol 1998;35:625-8.

17. Raihan AR, Zunaina E, Wan-Hazabbah WH, Adil H, Lakana-Kumar T. Neuroretinitis in ocular bartonellosis: a case series. Clin Opthalmol 2014;8:1459-66.

18. Habot-Wilner Z, Zur D, Goldstein M, Goldenberg D, Shulman S. Macular findings on optical coherence tomography in cat-scratch disease neuroretinitis. Eye (Lond) 2011; 25:1064-8

19. Eiger-Moscovich M, Amer R, Oray M, Tabbara KF, Tugal-Tutkun I, et al. Retinal artery occlusion due to Bartonella henselae infection: a case series. Acta Ophthalmol 2016;94:e367-70.

20. Vermeulen MJ, Verbakel H, Notermans DW, Reimerink JH, Peeters MF. Evaluation of sensitivity, specificity and cross-reactivity in Bartonella henselae serology. J Med Microbiol 2010;59:743-5.

21. La Scola B, Raoult D. Serological cross-reactions between Bartonella quintana, Bartonella henselae, and Coxiella burnetii. J Clin Microbiol 1996;34:2270-4.

22. Reed JB, Scales DK, Wong MT, Lattuada CP Jr, Dolan MJ, et al. Bartonella hensenlae neuroretinitis in cat scratch disease. Diagnosis, management, and sequelae. Opthalmology 1998;105:459-66. 